\title{
General criteria for quantum state smoothing with necessary and sufficient criteria for linear Gaussian quantum systems
}

\author{
Kiarn T. Laverick - Areeya Chantasri - Howard M. Wiseman
}

February 13, 2020

\begin{abstract}
Quantum state smoothing is a technique for estimating the quantum state of a partially observed quantum system at time $\tau$, conditioned on an entire observed measurement record (both before and after $\tau$ ). However, this smoothing technique requires an observer (Alice, say) to know the nature of the measurement records that are unknown to her in order to characterize the possible true states for Bob's (say) systems. If Alice makes an incorrect assumption about the set of true states for Bob's system, she will obtain a smoothed state that is suboptimal, and, worse, may be unrealizable (not corresponding to a valid evolution for the true states) or even unphysical (not represented by a state matrix $\rho \geq 0$ ). In this paper, we review the historical background to quantum state smoothing, and list general criteria a smoothed quantum state should satisfy. Then we derive, for the case of linear Gaussian quantum systems, a necessary and sufficient constraint for realizability on the covariance matrix of the true state. Naturally, a realizable covariance of the true state guarantees a smoothed state which is physical. It might be thought that any putative true covariance which gives a physical smoothed state would be a realizable true covariance, but we show explicitly that this is not so. This underlines the importance of the realizabilty constraint.
\end{abstract}

Centre for Quantum Computation and Communication Technology (Australian Research Council) . Centre for Quantum Dynamics, Griffith University, Nathan, Queensland 4111, Australia

E-mail: kiarn.laverick@griffithuni.edu.au

E-mail: a.chantasri@griffith.edu.au

E-mail: h.wiseman@griffith.edu.au

\section{Introduction}

Estimating the state of an open quantum system based on continuous-in-time measurement results is currently an important task in quantum science. Quantum state filtering [3, 4, also referred to as quantum trajectory theory, gives the optimal estimate of the quantum state based on the measurement record up until the estimation time. The quantum state smoothing theory of Ref. [13, on the other hand, estimates the quantum state based on an entire (both prior and posterior to the estimation time) measurement record. However, obtaining a valid smoothing theory, that is, a theory that results in a physical quantum state $\rho$, satisfying $\operatorname{Tr}[\rho]=1$ and $\rho \geq 0$ was not a trivial task $[2,22,21,12,7,13$.

In order to obtain physical smoothed quantum states, Guevara and Wiseman [13] considered a quantum system that is only partially observed by an observer. The state assigned by this observer, say Alice, will in general differ from the true state, i.e., the most accurate estimate of the quantum state assigned by an omniscient observer, say Bob. Bob is assumed to know two measurement records, one that is known to Alice ('observed' record) and one that is hidden to her ('unobserved' record). Even though Alice has no access to the unobserved record, she can still consider all possible unobserved records, and how likely each is, to calculate a smoothed quantum state.

For Alice to make an optimal smoothed estimate, she must know the type of measurement that led to the unobserved measurement record $[8$. However, she may not know this. In this work, we investigate whether it is possible for Alice to come up with some simple physical constraints on the possible true state, that limit the set of physical smoothed states, with minimal assumptions. This turns out to be possible for linear Gaus- 
sian quantum (LGQ) systems in a steady-state regime, where the covariance matrix of the LGQ state's Wigner function is deterministic. Consequently, we can derive a necessary and sufficient realizabilty constraint [26] for the set of realizable covariance matrices, assuming only that the unobserved measurement is fixed and diffusive in nature.

Given this new constraint on the true covariance, we investigate whether it is necessary for some putative true covariance to satisfy this condition in order to give, according to the smoothing formula we had previously derived [18, a covariance for the smoothed state which satisfies all uncertainty relations. We show that this is not the case. It is possible to select a putative true covariance that is unrealizable yet yields, naïvely following the procedure of Ref. [18, a smoothed state that is a mathematically allowable quantum state. Indeed, it turns out that even far more stringent tests on the reasonableness of the smoothed quantum state calculated using a putative true covariance cannot determine whether the latter satisfies the realizability constraint. This shows the importance of the realizability constraint we have derived in applying the theory of quantum state smoothing.

The structure of the paper is as follows. In Sec. 2 we provide some historical background and some general criteria for valid quantum state smoothing theories. In Sec. 3, we construct an argument following the Hughston-Josza-Wooters theorem in order to derive the necessary and sufficient realizability constraint on the true states for LGQ systems in steady state. Subsequently, in Sec. 4. we apply increasingly tighter constraints on the putative true states, culminating in the newly derived realizability constraint, to see how they affect the physicality of the putative smoothed state derived therefrom.

\section{History and General Criteria of Quantum State Smoothing}

In classical estimation theory, one is typically tasked with assigning a value to unknown parameters of a system, described by a vector $\mathbf{x}$, which we assume cannot be perfectly measured [20]. Given any information obtained from such a system, one of the most powerful tools at our disposal is a probability density function (PDF) $\wp(\mathbf{x})$ of the unknown, referred to as a state of the system. From this, one can calculate any type of estimator of $\mathbf{x}$, such as the mean or the mode of the distribution. However, in most cases, even the state of the system itself can be determined in different ways, and it is first necessary to specify upon what available measurement data the state $\wp(\mathbf{x} \mid C)$ is conditioned. Here
' $C$ ' refers to any conditioning on measurement records. This idea underpins the field of (classical) state estimation.

For a dynamical system under continuous observation, there exist optimal state estimation techniques, depending on the amount of measurement information available in time [20,11]. If the observed information is only available up until the time of estimation $\tau$, e.g. a real-time estimation, we can obtain a filtered estimate $\wp_{\mathrm{F}}(\mathbf{x}):=\wp(\mathbf{x} \mid \overleftarrow{\mathbf{O}})$ as a state conditioned on the 'past' measurement record $\overleftarrow{\mathbf{O}}=\left\{O_{t} ; t \in\left[t_{0}, \tau\right)\right\}$. The adjoint problem to the filtered state is the retrofiltered effect, commonly referred to as the likelihood function in the literature [11,6, 19], defined as $E_{\mathrm{R}}(\mathbf{x}):=\wp(\overrightarrow{\mathbf{O}} \mid \mathbf{x})$, where $\overrightarrow{\mathbf{O}}=\left\{O_{t} ; t \in[\tau, T)\right\}$. The effect tells us the likelihood of the 'future' measurement record $\overrightarrow{\mathbf{O}}$ given the system parameter $\mathbf{x}$ at time $\tau$. Finally, we can combine the filtered state and the retrofiltered effect to obtain a state conditioned on the entire, both past and future, measurement record $\overleftrightarrow{\mathbf{O}}=\left\{O_{t} ; t \in\left[t_{0}, T\right)\right\}$. This is known as the smoothed state, defined as $\wp_{\mathrm{S}}(\mathbf{x}):=\wp(\mathbf{x} \mid \overleftrightarrow{\mathbf{O}}) \propto$ $E_{\mathrm{R}}(\mathbf{x}) \wp_{\mathrm{F}}(\mathbf{x})$. Typically the smoothed state, when realtime estimation is not required, is more accurate [14 20.11 than the filtered state, as it is conditioned on more information.

When transitioning to quantum systems, we are concerned with estimating the quantum state of the system as it can be considered as a quantum analogue of the classical PDF. For example, we can calculate a mean estimator of any operator $\hat{A}$ from a quantum state $\rho$ via the expectation value $\langle\hat{A}\rangle=\operatorname{Tr}[\hat{A} \rho]$. By continuously monitoring the system, we can condition the evolution of the state on the past measurement record to obtain the filtered quantum state $\rho_{\mathrm{F}}$. This estimate of the state is sometimes called a quantum trajectory $3,4,25$. The quantum analog of the retrofiltered effect is the retrofiltered quantum effect $\hat{E}_{\mathrm{R}}$, a positive operator, defined such that $\wp(\overrightarrow{\mathbf{O}} \mid \rho)=\operatorname{Tr}\left[\hat{E}_{\mathrm{R}} \rho\right]$ is the likelihood function for a given $\rho$.

Following the analogy with the classical case, one could naïvely combine the two quantum operators, the filtered state and the retrofiltered effect, to obtain a 'smoothed' quantum operator,

$\varrho_{\mathrm{S}}=\frac{\hat{E}_{\mathrm{R}} \circ \rho_{\mathrm{F}}}{\operatorname{Tr}\left[\hat{E}_{\mathrm{R}} \circ \rho_{\mathrm{F}}\right]}$,

where we have used the Jordan product [16, 17] $A \circ B=$ $(A B+B A) / 2$ to symmetrize $\varrho_{\mathrm{S}}$, and have used the denominator $\operatorname{Tr}\left[\hat{E}_{\mathrm{R}} \circ \rho_{\mathrm{F}}\right]$ to normalise it. Unfortunately, the operator $\varrho_{\mathrm{S}}$ cannot properly represent a quantum state as one would hope. In general, this operator is not positive semidefinite, i.e., the criteria for a physical 
quantum state are not satisfied. Note that we are using a different notation $\varrho$ to distinguish it from a physical quantum state denoted with $\rho$. Interestingly, the trace of $\varrho_{\mathrm{S}}$ with an observable $\hat{A}$ gives,

$\operatorname{Tr}\left[\hat{A} \varrho_{\mathrm{S}}\right]=\frac{\operatorname{Tr}\left[\hat{E}_{\mathrm{R}} \hat{A} \rho_{\mathrm{F}}+\rho_{\mathrm{F}} \hat{A} \hat{E}_{\mathrm{R}}\right]}{2 \operatorname{Tr}\left[\hat{E}_{\mathrm{R}} \circ \rho_{\mathrm{F}}\right]}=\operatorname{Re}\left[\langle\hat{A}\rangle_{\mathrm{w}}\right]$,

which is not the usual expectation value of $\hat{A}$, but rather the real part of a complex weak value $\langle\hat{A}\rangle_{\mathrm{w}}$ that can have values outside the eigenvalue range of $\hat{A}$. The weak value was introduced in [1], where $\rho_{\mathrm{F}}$ and $\hat{E}_{\mathrm{R}}$ are to be interpreted as a generalized version of the pre- and postselected states in the two-state vector formalism [2, 23, 10. It is for this reason that this $\varrho_{\mathrm{S}}$ has been referred to as the smoothed weak-valued (SWV) state [18.

In a similar spirit as the SWV state, another formalism, called the past quantum state (PQS) [12, utilises the past-future measurement record in the form of the pair of operators $\Xi(\tau)=\left(\rho_{\mathrm{F}}, \hat{E}_{\mathrm{R}}\right)$ to estimate values of hidden results of any measurement performed at time $\tau$ on the quantum system. In the event that the measurement is weak, the estimated measurement reduce to the real part of the weak value, Eq. (2) 12 . Since $\Xi(\tau)$ itself comprises two operators, it is not a direct quantum analog of the classical smoothed state.

The above raises the question: what criteria should a theory of quantum state smoothing satisfy? Here we list four conditions:

(1) The theory should give a single smoothed quantum state $\rho_{\mathrm{S}}$ analogous to the classical state $\wp_{\mathrm{S}}$, and not a pair of states, for example.

(2) The smoothed state $\rho_{\mathrm{S}} \equiv \rho_{\overleftrightarrow{\mathrm{O}}}$ should reduce to its corresponding filtered state after averaging over all possible future measurement records given a past measurement record, i.e.,

$\rho_{\mathrm{F}} \equiv \rho_{\overleftarrow{\mathrm{O}}}=\sum_{\overrightarrow{\mathbf{O}}} \wp(\overrightarrow{\mathbf{O}} \mid \overleftarrow{\mathbf{O}}) \rho_{\mathrm{S}}$

(3) The smoothed quantum state should reduce to its classical counterpart when the initial conditions, final conditions, and dynamics of the system can all be described probabilistically in a fixed basis.

(4) The smoothed quantum state must be an S-class quantum state; that is, it must be Hermitian and positive semidefinite.

Formally, for a Hilbert space $\mathbb{H}$, the $\mathfrak{S}$-class quantum states are the set $\mathfrak{S}(\mathbb{H})=\{\rho \in \mathfrak{B}(\mathbb{H}): \rho \geq 0, \rho=$ $\left.\rho^{\dagger}, \operatorname{Tr}[\rho]=1\right\}$, where $\mathfrak{B}(\mathbb{H})$ is the set of bounded linear operators on $\mathbb{H}[15]$. We show, in Appendix A, that the SWV state satisfies the properties (1)-(3), but not (4). Moreover, it is worth noting that the properties (2) and
(4) imply that, if $\rho_{\mathrm{F}}$ is pure, then we have $\rho_{\mathrm{S}}=\rho_{\mathrm{F}}$. In other words, in order to obtain a non-trivial smoothed state, there must be missing information about the system. With these properties in mind, Guevara and Wiseman 13 devised a theory for quantum state smoothing, yielding a valid smoothed quantum state which satisfies all of the above propeties.

The quantum state smoothing theory [13] is defined for an open quantum system, using a scenario where the system is imperfectly monitored by an observer. As described in Fig. 1, the observer named Alice observes only her measurement record $\mathbf{O}$, whereas an omniscient observer, Bob, has access both to Alice's record and to the information Alice missed in her measurement, which comprises a measurement record $\mathbf{U}$ (that is unobserved by Alice). If Alice had access to the unobserved record, or equivalently made a perfect measurement on the system, she would have maximum knowledge about the quantum state and her estimated state would be the 'true' state $\rho_{\mathrm{T}} \equiv \rho_{\overleftarrow{\delta} \overleftarrow{\mathrm{U}}}$ which Bob has. Since Alice does not have access to the unobserved record, her task is to best estimate Bob's state using solely her observed record. Alice can define her estimated state as a conditioned state,

$\rho_{\mathrm{C}}=\sum_{\overleftarrow{\mathbf{U}}} \wp_{\mathrm{C}}(\overleftarrow{\mathbf{U}}) \rho_{\overleftarrow{\mathrm{O}} \overleftarrow{\mathbf{U}}}$

where the summation is over all possible past unobserved records $\overleftarrow{U}$ and the conditioning ' $\mathrm{C}$ ' can be any part of the observed record. For the case of filtering, $\rho_{\mathrm{C}}=\rho_{\mathrm{F}}$, the conditional probability distribution of the unobserved record becomes $\wp_{\mathrm{F}}(\overleftarrow{\mathbf{U}})=\wp(\overleftarrow{\mathbf{U}} \mid \overleftarrow{\mathbf{O}})$. In the same spirit, a proper smoothed quantum state is defined [13] by using the probability distribution of the unobserved record conditioned on the past-future measurement record, i.e., $\wp_{\mathrm{S}}(\overleftarrow{\mathbf{U}})=\wp(\overleftarrow{\mathbf{U}} \mid \overleftrightarrow{\mathbf{O}})$. As alluded to earlier (see Appendix A), this smoothed quantum state satisfies all of the properties (1)-(4) required for a quantum state smoothing theory, providing the first direct quantum analog of the classical smoothed state.

\section{Constraints on the underlying true states}

\subsection{General considerations}

While the quantum state smoothing theory satisfies all of the criteria mentioned earlier, it requires the unobserved unravelling performed by the secondary observer, Bob. Since Bob's record is unknown to Alice, it might seem like Bob's true state $\rho_{\overleftarrow{\mathbf{O}}, \overleftarrow{\mathbf{U}}}$ could be any arbitrary pure states of the system that still satisfies Eq. $\sqrt{4}$, for some $\wp \overleftrightarrow{\mathbf{O}}(\overleftarrow{\mathbf{U}})$. This idea is similar to the 


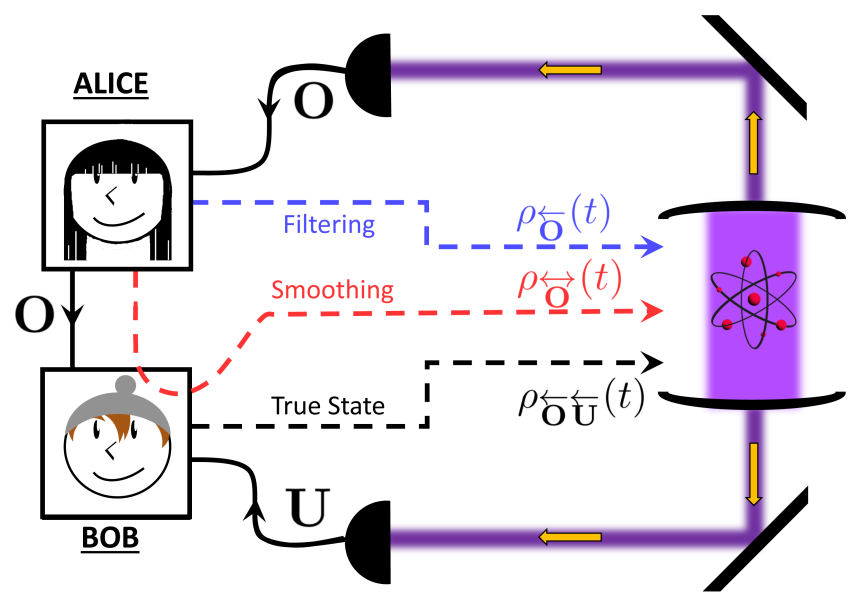

Fig. 1 A diagrammatic representation of the quantum state smoothing formalism. If Alice, who only has access to the observed record $\mathbf{O}$, wanted to assign a quantum state to the system, she could condition her estimate of the state on the past observed measurement record resulting in the filtered quantum state $\rho_{\mathrm{F}}:=\rho_{\overleftarrow{\mathrm{O}}}$. Another observer, Bob, can also assign a quantum state to the system conditioned on the his measurement record $\mathbf{U}$, in addition to the observed record, giving the true quantum state $\rho_{\mathrm{T}}:=\rho_{\overleftarrow{\mathrm{O}} \overleftarrow{\mathrm{U}}}$. At this point, if Alice knows that there is an unobserved measurement record, she can obtain a more accurate estimate of the true quantum state of the system by conditioning her estimate on the pastfuture observed measurement record to obtain the smoothed quantum state $\rho_{\mathrm{S}}:=\rho \overleftrightarrow{\mathrm{O}}$

fact that a mixed state can be written as a combination of pure states in infinitely many ways. However, as mooted in the Introduction, this is not the case. There are some physical constraints on Bob's possible true states to reflect the fact that the true state, in principle, is computed using an actual unobserved measurement record.

Let us restrict the discussion to a steady state of a monitored quantum system, where the system has evolved for a sufficiently long time and its dynamics are independent of any initial condition. In this case, a true state of the system will certainly be a pure state. The unconditional steady state is an ensemble average of true states $\rho_{\mathrm{T}}:=\rho_{\overleftarrow{\mathrm{O}} \overleftarrow{\mathrm{U}}}$ over both the past observed and unobserved measurement records,

$\rho^{\mathrm{ss}}=\sum_{\overleftarrow{\mathbf{O}}, \overleftarrow{\mathbf{U}}} \wp(\overleftarrow{\mathbf{O}}, \overleftarrow{\mathbf{U}}) \rho_{\mathrm{T}}$

Alice can condition her estimate of the quantum state on her past measurement record $\overleftarrow{\mathrm{O}}$ to obtain a filtered state

$\rho_{\mathrm{F}}=\sum_{\overleftarrow{\mathbf{U}}} \wp_{\mathrm{F}}(\overleftarrow{\mathbf{U}}) \rho_{\mathrm{T}}$,

where $\wp_{\mathrm{F}}(\overleftarrow{\mathbf{U}})=\wp(\overleftarrow{\mathbf{U}} \mid \overleftarrow{\mathbf{O}})$ as before. These two mixed states, both the unconditioned and filtered states in
Eqs. (5)-6), can be used to restrict the allowed true states by only considering the pure states that satisfies these equations. However, these constraints, even though necessary, are not sufficient for the realizable true states, as we now show.

The Hughston-Jozsa-Wooters (HJW) theorem provides a method for deriving an additional constraint. Consider a mixed quantum state of a steady state system, defined as

$\rho^{\mathrm{ss}}=\sum_{k} \wp_{k} \hat{\Pi}_{k}$

a mixture of pure states $\hat{\Pi}_{k}=\left|\psi_{k}\right\rangle\left\langle\psi_{k}\right|$ with probability weights $\wp_{k}>0$. In general, the pure states $\hat{\Pi}_{k}$ do not need to be orthogonal, and there are infinitely many ensembles $\left\{\wp_{k}, \hat{\Pi}_{k}\right\}_{k}$ that can represent the mixed state $\rho^{\mathrm{ss}}$. However, the HJW theorem states that, if a state is mixed solely because of its entanglement with an environment, it is possible to measure the environment in such a way that the system state collapses into a particular pure state $\hat{\Pi}_{k}$ in the ensemble with the corresponding probability $\wp_{k}$, without, on average, disturbing the system. Note, the measurement does not have to be a projective measurement, but can, for example, be a measurement at any time $t$ during a continuous monitoring of the system.

We follow Ref. 26] to utilise the HJW theorem for a continuously probed system in steady state. Given a system in a mixed unconditioned state $\rho^{\text {ss }}$, by measuring the environment at time $t$, the system's state collapses into one of the pure states $\hat{\Pi}_{k}$ of the ensemble $\left\{\wp_{k}, \hat{\Pi}_{k}\right\}_{k}$. After the measurement, the system reentangles with the environment for some time $\Delta t$, resulting in its state evolving into a mixed state $\rho(t+\Delta t)$ before the next measurement at time $t+\Delta t$. However, if we are to keep the same representation for the system, the measurement at time $t+\Delta t$ should collapse the system state to a pure state in the same ensemble. This is the condition for physically realizable states for continuous measurements, taking $\Delta t$ to be an infinitesimal time difference $\mathrm{d} t$.

In this work, we will adapt the physically realisability constraint derived in [26] to the Alice-Bob protocol, replacing the unconditioned state $\rho^{\mathrm{ss}}$ with Alice's filtered state, and the ensemble of pure states with the ensemble of Bob's possible true states. At some time $t$, Alice, with her observed record up to time $t$, assigns her best estimate of the state to be a mixed state $\rho_{\overleftarrow{\mathbf{O}}}(t)$. Bob, who can measure the environment unaccessible to Alice, collapses the system's state yielding a particular pure state in the ensemble of possible true states, given by Eq. (6), $\left\{\wp_{\mathrm{F}}(\overleftarrow{\mathbf{U}}), \rho_{\mathrm{T}}\right\}_{\overleftarrow{\mathrm{U}}}$. Evolving this state for some time dt only conditioning on Alice's measure- 
ment record, we obtain a new mixed state. Bob then, at time $t+\mathrm{d} t$, use his unobserved record for that time to collapse the state, again, into a possibly new true state.

At this point one might be tempted to just apply the analogous necessary condition derived in [26] by replacing the unconditioned state with the filtered state and the pure state with the true state, and be done with the problem. However, due to the stochastic nature of the filtered state as it is conditioned on the observed record, it is not a fixed state. As a consequence, we cannot, in general, claim that the system's mixed state at different time should have the same representation of true states. Nevertheless, a general constraint on the ensemble of physically realizable true states can derived for specific systems that have some level of determinism, where some properties of the filtered state remain unchanged throughout the evolution from time $t \rightarrow t+\mathrm{d} t$. An example of this is the class of LGQ systems [25, 9,24 to which we will restrict our discussion to henceforth.

\subsection{LGQ systems}

LGQ systems are continuous-variable quantum systems that can be described by $N$ bosonic modes, or equivalently a $2 N$ vector $\hat{\mathbf{x}}=\left(\hat{q}_{1}, \hat{p}_{1}, \ldots, \hat{q}_{N}, \hat{p}_{N}\right)^{\top}$, where $\hat{q}_{k}$ and $\hat{p}_{k}$ are the usual position and momentum operators satisfying $\left[\hat{q}_{k}, \hat{p}_{\ell}\right]=i \hbar \delta_{k \ell}$. As the name suggests, these systems have linear dynamics (in the sense defined below), and the Wigner representation of the quantum state is Gaussian, i.e., $W(\check{\mathbf{x}})=g(\check{\mathbf{x}} ;\langle\hat{\mathbf{x}}\rangle, V)$ with mean $\langle\hat{\mathbf{x}}\rangle$ and covariance $V$. The linearity constraint requires that the unconditioned Wigner function satisfies the Ornstein-Uhlenbeck equation 25]

$\dot{W}(\check{\mathbf{x}})=\left(-\nabla^{\top} A \check{\mathbf{x}}+\frac{1}{2} \nabla^{\top} D \nabla\right) W(\check{\mathbf{x}})$,

where $\nabla=\left(\partial / \partial x_{1}, \ldots, \partial / \partial x_{2 N}\right)^{\top}$ with $A$ and $D$ being constant matrices. Furthermore, it requires that any record $\mathbf{y}_{\mathrm{r}}$ resulting from a measurement of this LGQ system must be linear in $\hat{\mathbf{x}}$; that is $[25,9,24$

$\mathbf{y}_{\mathrm{r}} \mathrm{d} t=C_{\mathrm{r}}\langle\hat{\mathbf{x}}\rangle_{\mathrm{T}} \mathrm{d} t+\mathrm{d} \mathbf{w}_{\mathrm{r}}$

where $\langle\hat{\mathbf{x}}\rangle_{\mathrm{T}}$ is the true mean of the system, $C_{\mathrm{r}}$ is a constant matrix, $r \in\{\mathrm{o}, \mathrm{u}\}$, with ' $\mathrm{o}$ ' and ' $\mathrm{u}$ ' representing the observed and unobserved measurement records, respectively. Here, we have also introduced the measurement noise $d w_{r}$, a Weiner increment satisfying

$\mathbb{E}\left[\mathrm{d} \mathbf{w}_{\mathrm{r}}\right]=0, \quad \mathrm{~d} \mathbf{w}_{\mathrm{r}} \mathrm{d} \mathbf{w}_{\mathrm{r}^{\prime}}^{\top}=\delta_{\mathrm{rr}^{\prime}} I \mathrm{~d} t$

where $\mathbb{E}[\ldots]$ denotes an ensemble average and $I$ denotes the identity matrix. By satisfying these conditions above, it is guaranteed that the unconditioned and conditioned states remain Gaussian throughout their evolution.

To find the set of physically realizable true states, we begin with Alice. At time $t$, Alice computes and assigns her (mixed) filtered state, characterised by a filtered Wigner function $W_{\mathrm{F}}(\check{\mathbf{x}})=g\left(\check{\mathbf{x}} ;\langle\hat{\mathbf{x}}\rangle_{\mathrm{F}}, V_{\mathrm{F}}\right)$, to the system. The filtered mean $\langle\hat{\mathbf{x}}\rangle_{\mathrm{F}}$ and covariance $V_{\mathrm{F}}$, conditioned on the past observed record $\mathbf{y}_{\mathrm{o}}$, are given by [25, 18]

$\mathrm{d}\langle\hat{\mathbf{x}}\rangle_{\mathrm{F}}=A\langle\hat{\mathbf{x}}\rangle_{\mathrm{F}} \mathrm{d} t+\mathcal{K}_{\mathrm{o}}^{+}\left[V_{\mathrm{F}}\right] \mathrm{d} \mathbf{w}_{\mathrm{F}}$,

$\dot{V}_{\mathrm{F}}=A V_{\mathrm{F}}+V_{\mathrm{F}} A^{\top}+D-\mathcal{K}_{\mathrm{o}}^{+}\left[V_{\mathrm{F}}\right] \mathcal{K}_{\mathrm{o}}^{+}\left[V_{\mathrm{F}}\right]^{\top}$.

Here $\mathcal{K}_{\mathrm{o}}^{ \pm}[V]=V C_{\mathrm{o}}^{\top} \pm \Gamma_{\mathrm{o}}^{\top}$ and we have introduced the matrix $\Gamma_{\mathrm{o}}$ to account for the measurement back-action from the observed measurement record $\mathbf{y}_{\mathrm{o}}$. The stochastic nature of the mean can be seen in Eq. (11) through the vector of innovations $\mathrm{d} \mathbf{w}_{\mathrm{F}}=\mathbf{y}_{\mathrm{O}} \mathrm{d} t-C_{\mathrm{o}}\langle\hat{\mathbf{x}}\rangle_{\mathrm{F}} \mathrm{d} t$, which is a stochastic quantity describing the difference between the measurement result and the calculated estimate. On the other hand, the filtered covariance Eq. (12) is entirely deterministic and is fixed by the choice of $\mathrm{Al}$ ice's measurement unravelling [25, 9,24].

Since we are interested in the steady state, we assume that the eigenvalues of the drift matrix $A$ in Eq. (8) are negative and the time $t$ is sufficiently large that the system has reached its steady state. We can set the lefthand side of Eq. (12) to zero to solve for the steadystate solution $V_{\mathrm{F}}^{\mathrm{ss}}$. With Bob making the measurement unobserved by Alice, the state collapses into a particular pure state $W_{\mathrm{T}}(\check{\mathbf{x}})=g\left(\check{\mathbf{x}} ;\langle\hat{\mathbf{x}}\rangle_{\mathrm{T}}, V_{\mathrm{T}}^{\mathrm{ss}}\right)$ with mean $\langle\hat{\mathbf{x}}\rangle_{\mathrm{T}}$ and covariance $V_{\mathrm{T}}^{\mathrm{ss}}$. This true state will have a purity of unity, where purity for a Gaussian state is defined as $P=(\hbar / 2)^{N} \sqrt{\operatorname{det}\left(V^{-1}\right)}$ for a covariance $V$ [25]. To say that this state is one of the pure states in the mixture of Alice's filtered state, we require that

$V_{\mathrm{F}}^{\mathrm{ss}}-V_{\mathrm{T}}^{\mathrm{ss}} \geq 0$

meaning that $V_{\mathrm{T}}^{\mathrm{ss}}$ fits within $V_{\mathrm{F}}^{\mathrm{ss}}$ in $2 N$-dimensional phase space.

Following the scheme presented in the previous subsection for the physically realizable states, the state $W_{\mathrm{T}}(\check{\mathbf{x}})$ evolves with Eqs. 11 - 12 for a time $\mathrm{d} t$ to become a filtered state. The updated mean and variance of the new filtered state is given by

$$
\begin{array}{r}
\langle\hat{\mathbf{x}}\rangle_{\mathrm{F}}^{\prime}(t+\mathrm{d} t)=\langle\hat{\mathbf{x}}\rangle_{\mathrm{T}}(t)+\frac{A\langle\hat{\mathbf{x}}\rangle_{\mathrm{T}}(t) \mathrm{d} t+}{\mathcal{K}_{\mathrm{o}}^{+}\left[V_{\mathrm{F}}^{\prime}(t)\right] \mathrm{d} \mathbf{w}_{\mathrm{o}}(t),}
\end{array}
$$

$$
\begin{array}{r}
V_{\mathrm{F}}^{\prime}(t+\mathrm{d} t)=V_{\mathrm{F}}^{\prime}(t)+\left(A V_{\mathrm{F}}^{\prime}(t)+V_{\mathrm{F}}^{\prime}(t) A^{\top}+D-\right. \\
\left.\mathcal{K}_{\mathrm{o}}^{+}\left[V_{\mathrm{F}}^{\prime}(t)\right] \mathcal{K}_{\mathrm{o}}^{+}\left[V_{\mathrm{F}}^{\prime}(t)\right]^{\top}\right) \mathrm{d} t,
\end{array}
$$


where $V_{\mathrm{F}}^{\prime}(t)=V_{\mathrm{T}}^{\mathrm{ss}}$ and the prime is to distinguish this filtered mean and covariance from that in Eqs. (11)(12). Since this state at time $t+\mathrm{d} t$ is a filtered state, by definition, it is a mixture of true states at $t$. As a result, it must be the case that $V_{\mathrm{F}}^{\prime}(t+\mathrm{d} t)-V_{\mathrm{T}}^{\mathrm{ss}} \geq 0$, and from Eq. 15) we obtain

$A V_{\mathrm{T}}^{\mathrm{ss}}+V_{\mathrm{T}}^{\mathrm{ss}} A^{\top}+D-\mathcal{K}_{\mathrm{o}}^{+}\left[V_{\mathrm{T}}^{\mathrm{ss}}\right] \mathcal{K}_{\mathrm{o}}^{+}\left[V_{\mathrm{T}}^{\mathrm{ss}}\right]^{\top} \geq 0$.

This condition gives the set of realizable true covariances, which is the main result of this paper.

We can also show that Eq. 16 is a sufficient constraint for the true covariance by showing that a pure covariance that satisfies Eq. (16) must be the steadystate true covariance $V_{\mathrm{T}}^{\mathrm{ss}}$. Beginning with a pure true state with a Wigner function $W_{\mathrm{T}}(\check{\mathbf{x}})=g\left(\check{\mathbf{x}},\langle\hat{\mathbf{x}}\rangle_{\mathrm{T}}, V_{\mathrm{T}}\right)$ such that $V_{\mathrm{T}}$ that satisfies Eq. (16) at time $t$, the true state at an infinitesimal time later, $t+\mathrm{d} t$, when only conditioning on Alice's observed record, must evolve into a mixture of pure states, each with covariance $V_{\mathrm{T}}$ and Gaussian-distributed means. We know, by the HJW theorem, that Bob can measure the system in some way to collapse the system into one of the pure states in the ensemble with the appropriate probability and the resulting covariance of the collapsed pure state at time $t+\mathrm{d} t$ will be $V_{\mathrm{T}}$. Thus, Bob can always re-prepare a pure state with covariance $V_{\mathrm{T}}$ if he continuously monitors the system over the time interval $[t, t+\mathrm{d} t)$ and, consequently, since the covariance remains unchanged over the time interval, $V_{\mathrm{T}}$ must be the steady state solution $V_{\mathrm{T}}^{\mathrm{ss}}$ by definition. This means that Eq. (16) is necessary and sufficient for the true covariance in LGQ systems.

\subsection{Example: optical parametric oscillator}

We will now illustrate the effect of this constraint, Eq. 16p by considering a physical model, the on-threshold optical parametric oscillator (OPO) 25,24. The OPO system is described by the Lindblad master equation

$\hbar \dot{\rho}=-i[\hat{q} \hat{p}+\hat{p} \hat{q}, \rho]+\mathcal{D}[\hat{q}+i \hat{p}] \rho$,

where $\mathcal{D}[\hat{c}] \bullet=\hat{c} \bullet \hat{c}^{\dagger}-\left\{\hat{c}^{\dagger} \hat{c} / 2, \bullet\right\}$. This master equation yields $A=\operatorname{diag}(0,-2)$ and $D=\hbar I$, where $I$ is the $2 \times 2$ identity matrix. We also consider a homodyne measurement for Alice, giving $C_{\mathrm{o}}=2 \sqrt{\eta_{\mathrm{o}} / \hbar}\left(\cos \theta_{\mathrm{o}}, \sin \theta_{\mathrm{o}}\right)$ and $\Gamma_{\mathrm{o}}=-\hbar C_{\mathrm{o}} / 2$, where $\theta_{\mathrm{o}}$ and $\eta_{\mathrm{o}}$ are the homodyne phase of Alice's measurement and its efficiency. For this specific example we will consider $\theta_{\mathrm{o}}=3 \pi / 8$ and $\eta_{\mathrm{o}}=0.5$.

We consider four putative covariances for Bob, $V_{\mathrm{T}}^{\mathrm{ss}}=$ $V^{(i)}$ for $i \in\{a, b, c, d\}$. The specific covariance matrices are

$V^{(a)}=\frac{\hbar}{2}\left[\begin{array}{cc}2.41 & 0 \\ 0 & 0.41\end{array}\right], V^{(b)}=\frac{\hbar}{2}\left[\begin{array}{cc}3.18 & 0.49 \\ 0.49 & 0.39\end{array}\right]$,
$V^{(c)}=\frac{\hbar}{3}\left[\begin{array}{cc}5.02 & -0.50 \\ -0.50 . & 0.25\end{array}\right], V^{(d)}=\frac{\hbar}{2}\left[\begin{array}{ll}1.93 & 0.79 \\ 0.79 & 0.84\end{array}\right]$,

where the only covariance that satisfies the realizablility constraint Eq. 16 is $V^{(a)}$. Covariance $V^{(b)}$ was chosen to satisfy Eq. 113) and $V^{(c)}$ was chosen to satisfy

$V^{\mathrm{ss}}-V_{\mathrm{T}}^{\mathrm{ss}} \geq 0$

but not Eq. (13), which will be useful for the discussion in the next section. Finally, $V^{(d)}$ does not satisfy Eq. (19), but is still a pure state. In Fig. 2, we observe how these four states, with an initial mean $\langle\hat{\mathbf{x}}\rangle_{\mathrm{T}}=(0,0)^{\top}$, evolve under Eqs. 14 - 15. The only covariance that still fits within the evolved state is $V^{(a)}$, as expected since this satisfies Eq. (16). The remaining cases all have some region where the initial covariance is outside the evolved covariance (more detail is in the caption of Fig. 2).

\section{What the smoothed state reveals about physical realizability}

Now that we know the necessary and sufficient constraint for the true covariance to be physically realizable, the reader might be wondering whether a violation of this constraint can be detected directly from the smoothed quantum state. Explicitly, we ask the question: if Alice were told a putative true covariance $\tilde{V}_{\mathrm{T}}$ by Bob, and she used that, innocently, to calculate her smoothed state following the formulae in Ref. [18, would she be able to tell from the nature of that smoothed state whether the $\tilde{V}_{\mathrm{T}}$ she had been told could or could not be the actual true covariance.

Consider the same example as in the preceding section, Sec. 3.3 , the OPO system in Eq. 17), with the same parameters for the observed record as before $\left(\eta_{\mathrm{o}}=\right.$ $1 / 2$ and $\left.\theta_{\mathrm{o}}=3 \pi / 8\right)$, to help guide the analysis in this section. We can represent a putative true covariance as a $2 \times 2$ matrix,

$\tilde{V}_{\mathrm{T}}=\frac{\hbar}{2}\left[\begin{array}{ll}\alpha & \beta \\ \beta & \gamma\end{array}\right]$

Since the true state is pure, this will satisfy $\alpha \gamma-\beta^{2}=1$, so its parameters can be reduced to two, say $\gamma$ and $\delta=\beta / \sqrt{\alpha \gamma}[25$.

In Fig. 3 we plot, using the parameters $\gamma$ and $\delta$, a region in the parameter space of putative true state covariances, and the three conditions on it considered in Sec. 3.2 The weakest necessary condition is Eq. (19), meaning that the true state fits, in $2 N$-dimensional phase space, within the unconditioned steady state. Here 

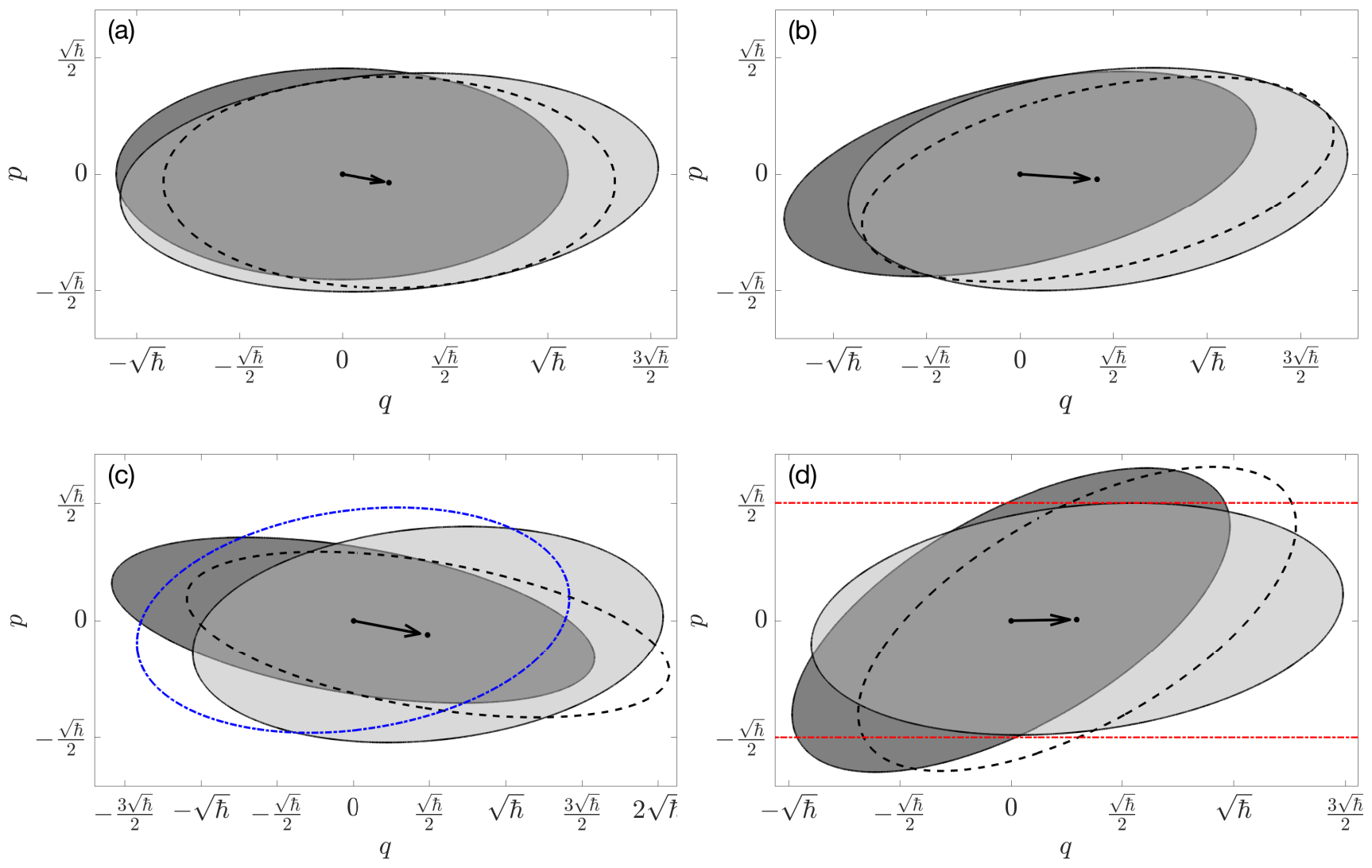

Fig. 2 The phase space diagrams for 1-SD contours of the Wigner functions for the OPO systems, showing the physical realisability condition for the Alice-Bob scenario. (See text for parameter details.) In all four graphs, (a)-(d), four cases are plotted with different $V_{\mathrm{T}}^{\mathrm{ss}}=V^{(i)}$ for $i \in\{a, b, c, d\}$, shown in Eq. 18 . The putative covariances $V_{\mathrm{T}}^{\mathrm{ss}}$ at time $t_{0}=0$ are plotted as the dark-grey ellipses, and are replotted as dashed ellipses with the means translated to their new values after evolving for a time $\Delta t=0.8 \mathrm{~s}$, in order to compare with the evolved covariances (light-grey ellipses) at the new time $t_{0}+\Delta t$ from Eq. (15). The mean values evolve according to Eq. 14. Only one case, (a), shows that the initial covariance fits inside its evolved state, because it satisfies Eq. (16). In the remaining cases, the covariances at $t_{0}+\Delta t$ have some regions outside their evolved states. For the cases (c) and (d), the initial covariances do not even fit within the filtered (blue dot-dashed) and the unconditioned (red dot-dashed) steady state covariances, respectively.

this corresponds to the constraint $\gamma<0.5$ (left of the dash-dot line). Within that region is the region (bounded by the dotted green line) of putative true states satisfying the stronger necessary condition is Eq. 13 , meaning that the true state fits within the steady-state filtered state. Within that region is the region (bounded by the dashed blue line) of putative true states satisfying the strongest necessary condition, also a sufficient condition, Eq. (16).

Now for quantum state smoothing, Alice calculates the covariance of her smoothed state, in steady state, directly from the putative true covariance via [18]

$V_{\mathrm{S}}^{\mathrm{ss}}=\left[\left(V_{\mathrm{F}}^{\mathrm{ss}}-V_{\mathrm{T}}^{\mathrm{ss}}\right)^{-1}+\left(V_{\mathrm{R}}^{\mathrm{ss}}+V_{\mathrm{T}}^{\mathrm{ss}}\right)^{-1}\right]^{-1}+V_{\mathrm{T}}^{\mathrm{ss}}$,

where the retrofiltered covariance $V_{\mathrm{R}}^{\mathrm{ss}}$ is the solution to the adjoint equation of Eq. (12), i.e., in the steady state,

$A V_{\mathrm{R}}^{\mathrm{ss}}+V_{\mathrm{R}}^{\mathrm{ss}} A^{\top}+D-\mathcal{K}_{\mathrm{o}}^{-}\left[V_{\mathrm{R}}^{\mathrm{ss}}\right] \mathcal{K}_{\mathrm{o}}^{-}\left[V_{\mathrm{R}}^{\mathrm{ss}}\right]^{\top}=0$.
The question is: does $V_{\mathrm{S}}^{\text {ss }}$ straight-fowwardly reveal whether $\tilde{V}_{\mathrm{T}}$ (and hence $V_{\mathrm{S}}^{\mathrm{ss}}$ ) is physically realizable?

To begin, we can look at when $V_{\mathrm{S}}^{\text {ss }}$ is an $\mathfrak{S}$-class state. For Gaussian systems, the necessary and sufficient criteria for an $\mathfrak{S}$-class state is that the covariance matrix $V$ satisfies the Schrödinger-Heisenberg uncertainty relation $V+i \hbar \Sigma / 2 \geq 0$ [25, 15, 5], where $\Sigma=$ $\oplus^{N}\left[\begin{array}{cc}0 & 1 \\ -1 & 0\end{array}\right]$. For $N=1$ (as here) this reduces to $\operatorname{det}(V) \geq$ $\hbar^{2} / 4$. In Fig. 3 we plot $\operatorname{det}\left(V_{\mathrm{S}}^{\mathrm{ss}}\right) /\left(\hbar^{2} / 4\right)$ for $V_{\mathrm{S}}^{\mathrm{ss}}$ as a function of the putative true state covariance parameters from Eq. 211. The $\tilde{V}_{\mathrm{T}}$ that give rise to $\mathfrak{S}$-class smoothed states are inside the solid black line. Clearly, the set of putative true covariances that result in $\mathfrak{S}$ class smoothed states is not restricted to the realizable true covariances as a good fraction (the area on the right side of the dot-dashed line) of these putative true covariances do not even satisfy Eq. (19). (Note however that here Eq. (13) is sufficient for the generated 
smoothed state to be $\mathfrak{S}$-class; see Appendix B for the proof that this is the case for all LGQ systems.)

One might wonder whether putting further restrictions on $V_{\mathrm{S}}^{\text {ss }}$ would help the situation. Since it is, by definition (4), a mixture of true states, $V_{\mathrm{S}}^{\mathrm{ss}}$ should also fit inside both the unconditioned state $V^{\mathrm{ss}}$ and the filtered state $V_{\mathrm{F}}^{\mathrm{ss}}$. That is Eq. (19) and Eq. (13) should both be satisfied with $V_{\mathrm{S}}^{\mathrm{ss}}$ in place of $\tilde{V}_{\mathrm{T}}$. Surprisingly, imposing these extra constraints makes no difference. That is, $V_{\mathrm{S}}^{\mathrm{sS}}$ by construction is guaranteed to satisfy these constraints even when $\tilde{V}_{\mathrm{T}}$ does not. This is proven in Appendix C for arbitrary LGQ systems. This is so because the equation for calculating the smoothed quantum state in Ref. [18] is oblivious to the unphysicality of the matrices which appear in it. Matrices which should be positive semidefinite, like $V_{\mathrm{F}}^{\mathrm{ss}}-\tilde{V}_{\mathrm{T}}^{\mathrm{ss}}$, may become indefinite without making the smoothed state obviously wrong. That is, Alice should not innocently accept a putative true covariance $\tilde{V}_{\mathrm{T}}^{\text {ss }}$ told to her by Bob, but should first check whether it satisfies the necessary and sufficient condition Eq. 16 .

In Fig. 3 we have also pointed out the four example putative true states considered in Sec. 3.3. As this shows, all four give rise to $\mathfrak{5}$-class smoothed states, even though only one satisfies Eq. (16). This, indicated by the triangle marker in Fig. 3 , corresponds to $V^{(a)}$ from Eq. (18). It results from a homodyne measurement by Bob with phase $\theta_{\mathrm{u}}=-\pi / 8$, and is an extremal point of the set of physically realizable $V_{\mathrm{T}}$. In fact the set of $V_{\mathrm{T}}$ generated by homodyne measurements for Bob, for all possible phases, forms the set of extremal points of the physically realizable set. (Note that this is not the case in general; it is a property of systems with a single mode $(N=1)$ and a single Lindblad operator $\hat{c})$. To illustrate this we have also considered a balanced heterodyne measurement [25] by Bob, indicated by the star marker, which is in the interior of the set of realizable covariances.

\section{Conclusion}

In this paper we reviewed the history that lead to the development of the quantum state smoothing theory [13, beginning from the classical notions of smoothing, and the motivation behind the theory. In formulating the smoothed quantum state, a true quantum state had to be introduced, which depends on a hidden measurement record. This motivated the investigation of the necessary constraints on the true state of the system when an observer has access to only the observed measurement record. A simple constraint is impossible to formulate general, as the evolution of the quantum system under observation is inherently stochastic

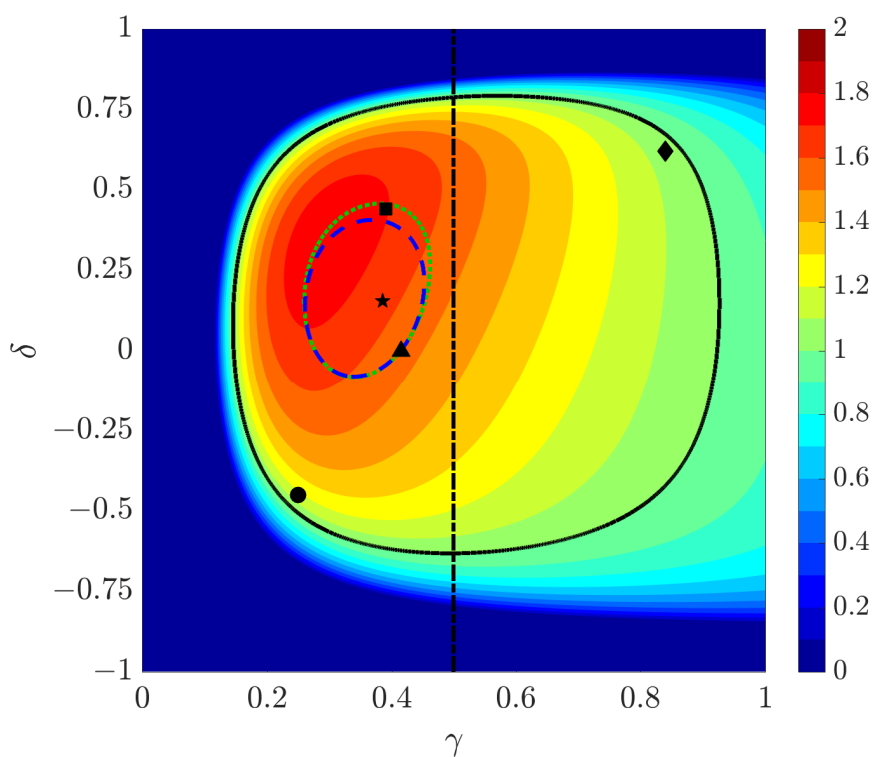

Fig. 3 A portion of the set of putative true covariances $\tilde{V}_{\mathrm{T}}$ in Eq. 201 , where we have defined $\delta=\beta / \sqrt{\alpha \gamma}$, for the example OPO system. (See text for parameter details.) The dotdashed line separates the covariances that satisfy Eq. 19p (area on the left). The green dotted and blue dashed lines indicates the covariances satisfying Eq. 13. and Eq. (16), respectively. The colours indicate the value of $(2 / \hbar)^{2} \operatorname{det}\left(V_{\mathrm{S}}^{\mathrm{SS}}\right)$ for the smoothed state covariance $V_{\mathrm{S}}^{\mathrm{ss}}$ calculated from $\tilde{V}_{\mathrm{T}}$ using the theory of Ref. [18. Values greater than one (S-class smoothed states) obtain inside the solid black line. The triangle, square, circle and diamond markers indicate the covariances matrices $V^{(a)}, V^{(b)}, V^{(c)}$ and $V^{(d)}$ in Eq. 18, respectively. We have also considered two particular unobserved unravellings for the system. The first is a homodyne unravelling with a phase $\theta_{\mathrm{u}}=-\pi / 8$, indicated by the triangle marker, and a balanced heterodyne unravelling indicated by the star marker.

and never reaches a steady state. However, for specific systems, like the LGQ systems we considered, there is enough determinism in the system to allow us to derive a necessary and sufficient constraint on the physically realizable true states. Finally, we find that the mathematical validity of a smoothed state calculated naïvely from a putative true covariance can not witness whether that covariance satisfied the physical realisability constraint. Nor do further, more stringent, conditions on the smoothed state, exemplifying the need for the realizability constraint.

An interesting area for future research would be to devise a method for calculating the optimal measurement strategy for Alice and Bob, resulting in the greatest increase in the purity of the smoothed state relative to that of the filtered state. It would also be useful to investigate how the effectiveness of quantum state smoothing is affected if the putative true state is physically realizable (i.e., corresponds to some unravelling by 
Bob), but the assumed unravelling by Bob was incorrect. Lastly, we have given (Sec. II) conditions under which quantum smoothing should reduce to classical smoothing, but it is an open question as to whether all of these conditions are necessary or whether they could be replaced by weaker conditions.

Acknowledgements We acknowledge the traditional owners of the land on which this work was undertaken at Griffith University, the Yuggera people. This research is funded by the Australian Research Council Centre of Excellence Program CE170100012. A.C. acknowledges the support of the Griffith University Postdoctoral Fellowship scheme.

\section{A Criteria for smoothed weak-value state and the smoothed quantum state}

In this appendix we will show how the SWV state and the smoothed quantum state satisfy the properties for a quantum state smoothing theory presented in Sec. 2

Property (1): The theory should give a single smoothed quantum state $\rho_{S}$ analogous to the classical state $\wp_{S}$, and not a pair of states, for example. It is obvious that the SWV state and the smoothed quantum state both satisfy this criterion by their respective definitions

$\varrho_{\mathrm{SWV}}=\frac{\hat{E}_{\mathrm{R}} \circ \rho_{\mathrm{F}}}{\operatorname{Tr}\left[\hat{E}_{\mathrm{R}} \circ \rho_{\mathrm{F}}\right]}$,
$\rho_{\mathrm{S}}=\sum_{\overleftarrow{\mathbf{U}}} \wp(\overleftarrow{\mathbf{U}} \mid \overleftrightarrow{\mathbf{O}}) \rho_{\overleftarrow{\mathrm{O}} \overleftarrow{\mathrm{U}}}$

Property (2): The smoothed state $\rho_{S} \equiv \rho_{\overleftrightarrow{\mathrm{o}}}$ should reduce to its corresponding filtered state after averaging over all possible future (observed) measurement records given a past measurement record. We will first consider the SWV state. Averaging the SWV state over the future measurement record given the past record gives

$$
\begin{aligned}
\sum_{\overrightarrow{\mathrm{O}}} \wp(\overrightarrow{\mathbf{O}} \mid \overleftarrow{\mathbf{O}}) \varrho_{\mathrm{S}} & =\sum_{\overrightarrow{\mathrm{O}}} \wp(\overrightarrow{\mathbf{O}} \mid \overleftarrow{\mathbf{O}}) \frac{\hat{E}_{\mathrm{R}} \circ \rho_{\mathrm{F}}}{\operatorname{Tr}\left[\hat{E}_{\mathrm{R}} \circ \rho_{\mathrm{F}}\right]} \\
& =\sum_{\overrightarrow{\mathrm{O}}} \hat{E}_{\mathrm{R}} \circ \rho_{\mathrm{F}},
\end{aligned}
$$

where we have used the fact that $\operatorname{Tr}\left[\hat{E}_{\mathrm{R}} \circ \rho_{\mathrm{F}}\right] \equiv \wp\left(\overrightarrow{\mathbf{O}} \mid \rho_{\mathrm{F}}\right)=$ $\wp(\overrightarrow{\mathbf{O}} \mid \overleftarrow{\mathbf{O}})$. By expanding the Jordan product and the completeness relationship for the effect,

$\sum_{\overrightarrow{\mathrm{O}}} \hat{E}_{\mathrm{R}}=\hat{1}$

where $\hat{1}$ is the identity operator, we obtain

$\sum_{\overrightarrow{\mathbf{O}}} \wp(\overrightarrow{\mathbf{O}} \mid \overleftarrow{\mathbf{O}}) \varrho_{\mathrm{S}}=\rho_{\mathrm{F}}$

Now for the smoothed quantum state, averaging over the future observed record conditioned on the past observed record gives

$$
\begin{aligned}
& \sum_{\overrightarrow{\mathrm{O}}} \wp(\overrightarrow{\mathbf{O}} \mid \overleftarrow{\mathbf{O}}) \rho_{\mathrm{S}}=\sum_{\overrightarrow{\mathrm{O}}} \wp(\overrightarrow{\mathbf{O}} \mid \overleftarrow{\mathbf{O}}) \sum_{\overleftarrow{\mathrm{U}}} \wp(\overleftarrow{\mathbf{U}} \mid \overleftrightarrow{\mathbf{O}}) \rho_{\overleftarrow{\mathrm{O}} \overleftarrow{\mathrm{U}}} \\
& =\sum_{\overleftarrow{\mathbf{U}}} \sum_{\overrightarrow{\mathbf{O}}} \wp(\overrightarrow{\mathbf{O}} \mid \overleftarrow{\mathbf{O}}) \wp(\overleftarrow{\mathbf{U}} \mid \overleftarrow{\mathbf{O}}, \overrightarrow{\mathbf{O}}) \rho_{\overleftarrow{\mathrm{O}} \overleftarrow{\mathrm{U}}} \\
& =\sum_{\overleftarrow{\mathbf{U}}} \sum_{\overrightarrow{\mathbf{O}}} \wp(\overrightarrow{\mathbf{O}} \mid \overleftarrow{\mathbf{O}}) \frac{\wp(\overleftarrow{\mathbf{U}}, \overrightarrow{\mathbf{O}} \mid \overleftarrow{\mathbf{O}})}{\wp(\overrightarrow{\mathbf{O}} \mid \overleftarrow{\mathbf{O}})} \rho_{\overleftarrow{\mathbf{O}} \overleftarrow{\mathrm{U}}} \\
& =\sum_{\overleftarrow{\mathbf{U}}} \wp(\overleftarrow{\mathbf{U}} \mid \overleftarrow{\mathbf{O}}) \rho_{\overleftarrow{\mathrm{O}} \overleftarrow{\mathrm{U}}} \equiv \rho_{\mathrm{F}}
\end{aligned}
$$

where we have used the definition of $\rho_{\mathrm{S}}$ and $\rho_{\mathrm{F}}$ in Eq. (4) and Bayes' theorem.

Property (3): The smoothed quantum state should reduce to its classical counterpart when the initial conditions, final conditions, and dynamics of the system can all be described probabilistically in a fixed basis. We assume that the filtered state and the retrofiltered effect are diagonal in a fixed orthonormal basis $\left|\psi_{x}\right\rangle$, which we can represent them as $\rho_{\mathrm{F}}=\sum_{x} \wp(x \mid \overleftarrow{\mathbf{O}})\left|\psi_{x}\right\rangle\left\langle\psi_{x}\right|$ and $\hat{E}_{\mathrm{R}}=\sum_{x^{\prime}} \wp\left(\overrightarrow{\mathbf{O}} \mid x^{\prime}\right)\left|\psi_{x^{\prime}}\right\rangle\left\langle\psi_{x^{\prime}}\right|$ respectively. To calculate the SWV state, assuming that the system is Markovian, i.e., satisfying $\wp\left(\overrightarrow{\mathbf{O}} \mid x^{\prime}\right) \equiv \wp\left(\overrightarrow{\mathbf{O}} \mid x^{\prime}, \overleftarrow{\mathbf{O}}\right)$, we can first compute

$$
\begin{aligned}
\rho_{\mathrm{F}} \hat{E}_{\mathrm{R}} & =\sum_{x, x^{\prime}} \wp(x \mid \overleftarrow{\mathbf{O}})_{\wp}\left(\overrightarrow{\mathbf{O}} \mid x^{\prime}\right)\left|\psi_{x}\right\rangle\left\langle\psi_{x} \mid \psi_{x^{\prime}}\right\rangle\left\langle\psi_{x^{\prime}}\right| \\
& =\sum_{x, x^{\prime}} \wp(x \mid \overleftarrow{\mathbf{O}})_{\wp}\left(\overrightarrow{\mathbf{O}} \mid x^{\prime}, \overleftarrow{\mathbf{O}}\right) \delta_{x x^{\prime}}\left|\psi_{x}\right\rangle\left\langle\psi_{x^{\prime}}\right| \\
& =\sum_{x} \wp(x \mid \overleftrightarrow{\mathbf{O}}) \wp(\overrightarrow{\mathbf{O}} \mid \overleftarrow{\mathbf{O}})\left|\psi_{x}\right\rangle\left\langle\psi_{x}\right|
\end{aligned}
$$

It is easy to see that the reverse ordering $\hat{E}_{\mathrm{R}} \rho_{\mathrm{F}}$ gives the same result, and we can calculate the SWV state

$$
\begin{aligned}
\varrho_{\mathrm{SWV}} & =\frac{\sum_{x} \wp(x \mid \overleftrightarrow{\mathbf{O}}) \wp(\overrightarrow{\mathbf{O}} \mid \overleftarrow{\mathbf{O}})\left|\psi_{x}\right\rangle\left\langle\psi_{x}\right|}{\operatorname{Tr}\left[\hat{E}_{\mathrm{R}} \circ \rho_{\mathrm{F}}\right]} \\
& =\sum_{x} \wp(x \mid \overleftrightarrow{\mathbf{O}})\left|\psi_{x}\right\rangle\left\langle\psi_{x}\right|,
\end{aligned}
$$

where $\operatorname{Tr}\left[\hat{E}_{\mathrm{R}} \circ \rho_{\mathrm{F}}\right]=\wp(\overrightarrow{\mathbf{O}} \mid \overleftarrow{\mathbf{O}})$. We can see that the SWV state has reduced to the classical smoothed state $\wp_{\mathrm{S}}(x)=\wp(x \mid \overleftrightarrow{\mathbf{O}})$

We will now show that the smoothed quantum state also reduces to the classical smoothed state when the true state is diagonal in a fixed orthonormal basis. Firstly, we note that we can represent the true state by

$\rho_{\overleftarrow{\mathrm{O}} \overleftarrow{\mathrm{U}}}=\sum_{x} \wp(x \mid \overleftarrow{\mathbf{O}}, \overleftarrow{\mathbf{U}})\left|\psi_{x}\right\rangle\left\langle\psi_{x}\right|$

We also notice that $\wp(x \mid \overleftarrow{\mathbf{O}}, \overleftarrow{\mathbf{U}})=\wp(x \mid \overleftrightarrow{\mathbf{O}}, \overleftarrow{\mathbf{U}})$, since the true state contains the maximum information about the system, and conditioning the system on anymore information cannot influence the state. By taking this into consideration, we can write the smoothed quantum state as

$$
\begin{aligned}
\rho_{\mathrm{S}} & =\sum_{\overleftarrow{\mathbf{U}}} \wp(\overleftarrow{\mathbf{U}} \mid \overleftrightarrow{\mathbf{O}}) \sum_{x} \wp(x \mid \overleftrightarrow{\mathbf{O}}, \overleftarrow{\mathbf{U}})\left|\psi_{x}\right\rangle\left\langle\psi_{x}\right| \\
& =\sum_{x} \sum_{\overleftarrow{\mathbf{U}}} \wp(\overleftarrow{\mathbf{U}} \mid \overleftrightarrow{\mathbf{O}}) \wp(x \mid \overleftrightarrow{\mathbf{O}}, \overleftarrow{\mathbf{U}})\left|\psi_{x}\right\rangle\left\langle\psi_{x}\right| \\
& =\sum_{x} \wp(x \mid \overleftrightarrow{\mathbf{O}})\left|\psi_{x}\right\rangle\left\langle\psi_{x}\right|
\end{aligned}
$$


Property (4): The smoothed quantum state must alway be Hermitian and positive semidefinite, that is, it must be a $\mathfrak{S}$-class quantum state. Beginning with the SWV state, we can see that this operator is Hermitian by taking the Hermitian conjugate

$\varrho_{\mathrm{S}}^{\dagger}=\frac{\hat{E}_{\mathrm{R}} \circ \rho_{\mathrm{F}}}{\operatorname{Tr}\left[\hat{E}_{\mathrm{R}} \circ \rho_{\mathrm{F}}\right]}=\varrho_{\mathrm{S}}$,

since $\hat{E}_{\mathrm{R}}^{\dagger}=\hat{E}_{\mathrm{R}}$. However, the SWV state cannot be guaranteed to be positive semidefinite. The product of two positive semidefinite operators $\rho_{\mathrm{F}}$ and $\hat{E}_{\mathrm{R}}$ is only guaranteed to be positive semidefinite if they commute, which is not necessarily the case. For the smoothed quantum state, it is clear that the state is both Hermitian and positive semidefinite as it is a mixture of true quantum states, which must be Hermitian and positive semidefinite.

\section{B Proof that Eq. (13) is sufficient to generate an S-class smoothed state}

For the smoothed state to be an $\mathfrak{S}$-class state, it must satisfy: $V_{\mathrm{S}}+i \hbar \Sigma / 2 \geq 0$. Considering a putative true covariance $\tilde{V}_{\mathrm{T}}$ and the smoothed covariance in Eq. 21, we would like to show that

$\left[\left(V_{\mathrm{F}}-\tilde{V}_{\mathrm{T}}\right)^{-1}+\left(V_{\mathrm{R}}+\tilde{V}_{\mathrm{T}}\right)^{-1}\right]^{-1}+\tilde{V}_{\mathrm{T}}+\frac{i \hbar}{2} \Sigma \geq 0$,

is true for any $\mathfrak{S}$-class $\tilde{V}_{\mathrm{T}}$ that satisfies Eq. 13 . Since $\tilde{V}_{\mathrm{T}}$ is an $\mathfrak{S}$-class state, it must satisfy $\tilde{V}_{\mathrm{T}}+i \hbar \Sigma / 2 \geq 0$ and as a result, for $V_{\mathrm{S}}$ to be an $\mathfrak{S}$-class state, we only require that

$\left[\left(V_{\mathrm{F}}-\tilde{V}_{\mathrm{T}}\right)^{-1}+\left(V_{\mathrm{R}}+\tilde{V}_{\mathrm{T}}\right)^{-1}\right]^{-1} \geq 0$.

It is always the case that $\left(V_{\mathrm{R}}+\tilde{V}_{\mathrm{T}}\right)^{-1} \geq 0$ since $V_{\mathrm{R}}$ and $\tilde{V}_{\mathrm{T}}$ are individually positive semidefinite and, by assumption, it is that case that $V_{\mathrm{F}}-\tilde{V}_{\mathrm{T}} \geq 0$. Consequently, provided the necessary inverses exist, Eq. 44 is true for any putative true covariance that satisfies Eq. (13). Thus the smoothed state be an $\mathfrak{S}$-class quantum state.

\section{Proof that the smoothed covariance always fits within the filtered covariance}

We want to show that $V_{\mathrm{F}}-V_{\mathrm{S}} \geq 0$ for any putative true state $\tilde{V}_{\mathrm{T}}$. To begin, consider

$$
\begin{aligned}
V_{\mathrm{F}}-V_{\mathrm{S}}= & V_{\mathrm{F}}-\left[\left(V_{\mathrm{F}}-\tilde{V}_{\mathrm{T}}\right)^{-1}+\left(V_{\mathrm{R}}+\tilde{V}_{\mathrm{T}}\right)^{-1}\right]^{-1}-V_{\mathrm{T}} \\
= & \left(V_{\mathrm{F}}-\tilde{V}_{\mathrm{T}}\right)-\left(V_{\mathrm{F}}-\tilde{V}_{\mathrm{T}}\right) \times \\
& {\left[I+\left(V_{\mathrm{R}}+\tilde{V}_{\mathrm{T}}\right)^{-1}\left(V_{\mathrm{F}}-\tilde{V}_{\mathrm{T}}\right)\right]^{-1} } \\
= & \left(V_{\mathrm{F}}-\tilde{V}_{\mathrm{T}}\right)-\left(V_{\mathrm{F}}-\tilde{V}_{\mathrm{T}}\right) \times \\
& \left\{I-\left[I+\left(V_{\mathrm{R}}+\tilde{V}_{\mathrm{T}}\right)^{-1}\left(V_{\mathrm{F}}-\tilde{V}_{\mathrm{T}}\right)\right]^{-1} \times\right. \\
& \left.\left(V_{\mathrm{R}}+\tilde{V}_{\mathrm{T}}\right)^{-1}\left(V_{\mathrm{F}}-\tilde{V}_{\mathrm{T}}\right)\right\} \\
= & \left(V_{\mathrm{F}}-\tilde{V}_{\mathrm{T}}\right)\left[I+\left(V_{\mathrm{R}}+\tilde{V}_{\mathrm{T}}\right)^{-1}\left(V_{\mathrm{F}}-\tilde{V}_{\mathrm{T}}\right)\right]^{-1} \times \\
& \left(V_{\mathrm{R}}+\tilde{V}_{\mathrm{T}}\right)^{-1}\left(V_{\mathrm{F}}-\tilde{V}_{\mathrm{T}}\right),
\end{aligned}
$$

where in Eq. (47) we have used the identity $(I+P)^{-1}=$ $I-(I+P)^{-1} P$. Since $\left(V_{\mathrm{F}}-\tilde{V}_{\mathrm{T}}\right)$ is symmetric, $V_{\mathrm{F}}-V_{\mathrm{S}}$ will be positive semidefinite if

$\left[I+\left(V_{\mathrm{R}}+\tilde{V}_{\mathrm{T}}\right)^{-1}\left(V_{\mathrm{F}}-\tilde{V}_{\mathrm{T}}\right)\right]^{-1}\left(V_{\mathrm{R}}+\tilde{V}_{\mathrm{T}}\right)^{-1} \geq 0$.
Now,

$$
\begin{aligned}
& {\left[I+\left(V_{\mathrm{R}}+\tilde{V}_{\mathrm{T}}\right)^{-1}\left(V_{\mathrm{F}}-\tilde{V}_{\mathrm{T}}\right)\right]^{-1}\left(V_{\mathrm{R}}+\tilde{V}_{\mathrm{T}}\right)^{-1}} \\
& =\left[\left(V_{\mathrm{R}}+\tilde{V}_{\mathrm{T}}\right)\left\{I+\left(V_{\mathrm{R}}+\tilde{V}_{\mathrm{T}}\right)^{-1}\left(V_{\mathrm{F}}-\tilde{V}_{\mathrm{T}}\right)\right\}\right]^{-1} \\
& =\left[V_{\mathrm{R}}+V_{\mathrm{T}}+V_{\mathrm{F}}-V_{\mathrm{T}}\right]^{-1} \\
& =\left[V_{\mathrm{R}}+V_{\mathrm{F}}\right]^{-1} \geq 0,
\end{aligned}
$$

where the last line follows from the fact that $V_{\mathrm{F}} \geq 0$ and $V_{\mathrm{R}} \geq 0$. Hence, we have proven that for any putative true state $\tilde{V}_{\mathrm{T}}$ that $V_{\mathrm{F}}-V_{\mathrm{S}} \geq 0$. From this we can also show, trivially, that $V^{\mathrm{ss}}-V_{\mathrm{S}} \geq 0$ since $V^{\mathrm{ss}}-V_{\mathrm{F}} \geq 0$, i.e.,

$V^{\mathrm{ss}}-V_{\mathrm{S}}=\left(V^{\mathrm{ss}}-V_{\mathrm{F}}\right)+\left(V_{\mathrm{F}}-V_{\mathrm{S}}\right) \geq 0$.

\section{References}

1. Aharonov, Y., Albert, D.Z., Vaidman, L.: How the result of a measurement of a component of the spin of a spin- $1 / 2$ particle can turn out to be 100 . Phys. Rev. Lett. 60, 1351-1354 (1988). DOI 10.1103/ PhysRevLett.60.1351. URL https://link.aps.org/doi/ 10.1103/PhysRevLett.60.1351

2. Aharonov, Y., Bergmann, P.G., Lebowitz, J.L.: Time symmetry in the quantum process of measurement. Phys. Rev. 134, B1410-B1416 (1964). DOI 10.1103/PhysRev. 134.B1410. URL https://link.aps.org/doi/10.1103/ PhysRev.134.B1410

3. Belavkin, V.P.: Information, complexity and control in quantum physics. Springer, New York (1987)

4. Belavkin, V.P.: Quantum continual measurements and a posteriori collapse on ccr. Communications in Mathematical Physics 146(3), 611-635 (1992). DOI 10.1007/ BF02097018. URL https://doi.org/10.1007/BF02097018

5. Braunstein, S.L., van Loock, P.: Quantum information with continuous variables. Rev. Mod. Phys. 77, 513-577 (2005). DOI 10.1103/RevModPhys.77.513. URL https: //link.aps.org/doi/10.1103/RevModPhys.77.513

6. Brown, R.G., Hwang, P.Y.C.: Introduction to Random Signals and Applied Kalman Filtering, 4 edn. Wiley, New York (2012)

7. Chantasri, A., Dressel, J., Jordan, A.N.: Action principle for continuous quantum measurement. Phys. Rev. A 88, 042110 (2013)

8. Chantasri, A., Guevara, I., Wiseman, H.M.: Quantum state smoothing: why the types of observed and unobserved measurements matter. New Journal of Physics 21(8), 083039 (2019). DOI 10.1088/1367-2630/ab396e. URL https ://doi.org/10.1088\%2F1367-2630\%2Fab396e

9. Doherty, A.C., Jacobs, K.: Feedback control of quantum systems using continuous state estimation. Phys. Rev. A 60(4), 2700 (1999). DOI 10.1103/PhysRevA.60.2700

10. Dressel, J., Agarwal, S., Jordan, A.N.: Contextual values of observables in quantum measurements. Physical review letters 104(24), 240401 (2010). DOI 10.1103/ PhysRevLett.104.240401

11. Einicke, G.A.: Smoothing, filtering and prediction: Estimating the past, present and future. InTech Rijeka (2012)

12. Gammelmark, S., Julsgaard, B., Mølmer, K.: Past Quantum States of a Monitored System. Phys. Rev. Lett. 111, 160401 (2013)

13. Guevara, I., Wiseman, H.: Quantum state smoothing. Phys. Rev. Lett. 115, 180407 (2015). DOI 10.1103/ PhysRevLett.115.180407. URL http://link.aps.org/ doi/10.1103/PhysRevLett.115.180407 
14. Haykin, S.: Kalman Filtering and Neural Networks. Wiley, New York (2001)

15. Holevo, A.S.: Probabilistic and Statistical Aspects of Quantum Theory, Statistics and Probability, vol. 1. NorthHolland, Amsterdam (1982)

16. Jordan, P.: Über die multiplikation quantenmechanischer größen. Zeitschrift für Physik 80(5-6), 285-291 (1933)

17. Jordan, P., v. Neumann, J., Wigner, E.: On an algebraic generalization of the quantum mechanical formalism. Annals of Mathematics 35(1), 29-64 (1934). URL http://www.jstor.org/stable/1968117

18. Laverick, K.T., Chantasri, A., Wiseman, H.M.: Quantum state smoothing for linear gaussian systems. Phys. Rev. Lett. 122, 190402 (2019). DOI 10.1103/PhysRevLett. 122.190402. URL https://link.aps.org/doi/10.1103/ PhysRevLett.122.190402

19. Särkkä, S.: Bayesian filtering and smoothing, vol. 3. Cambridge University Press (2013)

20. Trees, H.L.V., Bell, K.L.: Detection, Estimation, and Modulation Theory, Part I: Detection, Estimation, and Filtering Theory, 2 edn. John Wiley and Sons, New York (2013)

21. Tsang, M.: Optimal waveform estimation for classical and quantum systems via time-symmetric smoothing. Phys. Rev. A 80(3), 033840 (2009). DOI 10.1103/PhysRevA. 80.033840

22. Tsang, M.: Time-symmetric quantum theory of smoothing. Phys. Rev. Lett. 102(25), 250403 (2009). DOI 10.1103/PhysRevLett.102.250403

23. Wiseman, H.M.: Weak values, quantum trajectories, and the cavity-qed experiment on wave-particle correlation. Physical Review A 65(3), 032111 (2002). DOI 10.1103/ PhysRevA.65.032111

24. Wiseman, H.M., Doherty, A.C.: Optimal unravellings for feedback control in linear quantum systems. Phys. Rev. Lett. 94(7), 070405 (2005). DOI 10.1103/PhysRevLett. 94.070405

25. Wiseman, H.M., Milburn, G.J.: Quantum Measurement and Control. Cambridge University Press, Cambridge, England (2010)

26. Wiseman, H.M., Vaccaro, J.A.: Inequivalence of pure state ensembles for open quantum systems: The preferred ensembles are those that are physically realizable. Phys. Rev. Lett. 87(24), 240402 (2001). DOI 10.1103/PhysRevLett.87.240402 